\title{
Real-World Therapeutic Outcomes of S-1 Adjuvant Chemotherapy for pStage II/III Gastric Cancer in the Elderly
}

\author{
Chikara Kunisaki $^{a}$ Sho Sato $^{a}$ Nobuhiro Tsuchiya ${ }^{a}$ Hirokazu Kubo $^{a}$ \\ Jun Watanabe ${ }^{a}$ Tsutomu Sato ${ }^{a}$ Kazuhisa Takeda ${ }^{a}$ Yuko Tamura ${ }^{b}$ \\ Kohei Kasahara $^{\mathrm{b}}$ Takashi Kosaka $^{\mathrm{b}}$ Hirotoshi Akiyama ${ }^{\mathrm{b}}$ Itaru Endo ${ }^{\mathrm{b}}$ \\ ${ }^{a}$ Department of Surgery, Gastroenterological Center, Yokohama City University, Yokohama, Japan; \\ ${ }^{b}$ Department of Gastroenterological Surgery, Yokohama City University, School of Medicine, Yokohama, Japan
}

\section{Keywords}

Gastric cancer · S-1 · Adjuvant chemotherapy · Elderly ·

Overall survival $\cdot$ Relapse-free survival

\begin{abstract}
Background: The predictive factors for discontinuation of S-1 administration and prognostic factors in elderly patients with pStage II/III gastric cancer receiving S-1 adjuvant chemotherapy remain unclear. Methods: Between January 2004 and December 2016, 80 elderly gastric cancer patients ( $\geq 70$ years) undergoing curative D2 gastrectomy were enrolled in this study. Predictive factors for completion of S-1 administration over 1 year, adverse events due to S-1 administration, and prognostic factors for overall survival (OS) and relapsefree survival (RFS) were evaluated. Results: Twenty-eight patients (35\%) completed 8 courses of S-1. The median relative dose intensity was $82.1 \%$ (IQR 31.1-100\%). The incidence rates of hematological and nonhematological adverse events were acceptable. Distal gastrectomy was an independent predictive factor for completion of S-1 administration (odds ratio [OR] 0.364; 95\% confidence interval [CI] 0.141-
\end{abstract}

karger@karger.com www.karger.com/esr

Karger $\stackrel{\text { ' }}{5}$

BOPEN ACCESS
(C) 2021 The Author(s)

Published by S. Karger AG, Basel

This is an Open Access article licensed under the Creative Common Attribution-NonCommercial-4.0 International License (CC BY-NC) (http://www.karger.com/Services/OpenAccessLicense), applicable to the online version of the article only. Usage and distribution for commercial purposes requires written permission.
0.939; $p=0.037)$. Higher postoperative neutrophil count/ lymphocyte count (N/L) ratio and more advanced stage adversely influenced OS. Multivariate analysis revealed that a higher postoperative N/L ratio and more advanced stage adversely affected RFS. Conclusion: To complete adjuvant S-1 administration to elderly patients with pStage II/III gastric cancer, total gastrectomy should be avoided if possible. A new regimen for elderly gastric cancer patients with higher postoperative $\mathrm{N} / \mathrm{L}$ ratios and more advanced stage should be established.

(c) 2021 The Author(s)

Published by S. Karger AG, Basel

\section{Introduction}

Although the incidence of gastric cancer has been decreasing, this disease remains the fifth leading cause of cancer-related death in the world $[1,2]$. The incidence of gastric cancer is still high in East Asia. The therapeutic strategy for early gastric cancer has been varied, and therapeutic outcomes have been satisfactory in recent decades $[3,4]$. However, the therapeutic outcomes of ad- 
vanced gastric cancer remain unsatisfactory [5]. To improve these outcomes, a Japanese phase III trial of adjuvant chemotherapy, the Adjuvant Chemotherapy Trial of TS- 1 for Gastric Cancer (ACTS-GC), was conducted for pStage II and IIIA/B gastric cancer patients undergoing D2 gastrectomy $[6,7]$. This trial showed that S-1 administration for 12 months provided significant survival benefit in pStage II and pStage III after R0 gastrectomy compared with same-stage patients treated by surgery only. However, it is difficult to complete S-1 administration over 12 months in some patients. In the early phase after gastrectomy, destruction of the original anatomy of the stomach induces reflux, the small or absent remnant of the stomach reduces the volume of oral food intake, and the insufficiency of digestive and absorption function induces malabsorption. Therefore, it is difficult to continue planned administration of S-1 to patients with some specific factors. As the ACTS-GC study showed the efficacy of continuation of S-1 for 12 months (8 courses) and a noninferiority study (JCOG 1104) showed the superiority of 8 courses of S-1, it is mandatory to identify the predictive factors for continuation of S-1 administration [8].

The efficacy of treatments using S-1 for several cancers has been reported all over the world. The promising effects of S-1 were shown by the following trials: a phase 3 trial including S-1 for gastric cancer [9], immune checkpoint inhibitor combination with S-1 for gastric cancer [10], and doublet treatments according to histological type for gastric cancer [11]; newly developed TAS-114 with combination with S-1 for non-small-cell lung cancer [12]; and oxaliplatin plus S-1 combination chemotherapy for older vulnerable patients with colorectal cancer [13]. It is likely that the clinical usefulness of S-1 will be demonstrated more and more in various cancers all over the world.

However, several studies have reported that age, performance status, renal dysfunction, body weight loss, and postoperative complications affect the planned continuation of adjuvant S-1 $[14,15]$. We frequently encounter elderly patients with pStage II/III gastric cancer who suffer from the adverse events of $S-1$. We are concerned about poor long-term survival due to discontinuation of S-1 in the elderly.

It is important to identify the real-world predictive factors for discontinuation of S-1 administration and determine prognostic factors for overall survival (OS) and relapse-free survival (RFS) in elderly patients with pStage II/III gastric cancer at a high-volume center to obtain favorable long-term outcomes. We evaluated the predictive factors for the discontinuation of S-1 and prognostic factors for the long-term survival of elderly patients undergoing R0 gastrectomy.

\section{Materials and Methods}

\section{Patients}

Between January 2004 and December 2016, at the Department of Surgery, Gastroenterological Center, Yokohama City University, Japan, 218 elderly gastric adenocarcinoma patients $(\geq 70$ years) who underwent D2 curative gastrectomy (R0) were diagnosed as having pStage II or III (IIIA and IIIB). Adjuvant S-1 was administered to 80 patients $(36.7 \%)$. Initiation of oral administration of S-1 was attempted within 6 weeks after surgery. S-1 (40 mg/ $\mathrm{m}^{2}$ ) was administered to most of the patients twice daily for 4 weeks followed by 2 weeks of rest. Six weeks of S- 1 administration was regarded as 1 course, and 8 courses continued for 1 year if there were no patient refusals, uncontrollable adverse events, or recurrences of cancer. According to the World Health Organisation (WHO), elderly patients are those $\geq 65$ years of age. In the ACTSGC trial [6], $24.2 \%$ of the patients were aged $\geq 70$ years; we therefore defined the elderly as $\geq 70$ years to obtain sufficient number of patients for analysis in this retrospective study.

Of 138 patients, 77 refused adjuvant chemotherapy and S-1 could not be administered to 61 due to their poor general condition after gastrectomy. The therapeutic outcomes of 80 patients were mainly analyzed in this study. OS and RFS were compared in the group that completed S-1 administration, the group with incomplete S-1 administration, the group that refused S-1 administration, and the group that did not receive S-1.

There were significant differences in age $(74.8 \pm 3.5$ vs. $77.5 \pm$ 4.8 years, $p<0.0001$ ), ASA score (grade 1/2/3: 27/39/14 vs. $22 / 65 / 51, p<0.001$ ), and postoperative complications (presence/ absence of Clavien-Dindo $\geq$ grade III: $20 / 60$ vs. $54 / 84, p=0.038$ ) between the S-1 administered group and the nonadministered group, respectively. Patients in the nonadministered group were older and had worse a preoperative general condition and a higher incidence of postoperative complications. However, sex, type of operation, and stage did not differ between the 2 groups.

All patients were diagnosed as having gastric adenocarcinoma by using imaging techniques and endoscopic biopsies. They also underwent barium swallow tests in conjunction with computed tomography (CT) scans to identify the site of the tumor precisely and determine the operative method prior to surgery. Ultrasonography of the abdomen was also performed when abnormal findings were detected in the liver, gall bladder, or pancreas on CT scans.

Data on patient characteristics, laboratory findings, and surgical methods of gastrectomy were obtained from the database. Tumor stage was based on the Japanese Classification of Gastric Carcinoma (2nd English ed.) [16] because ACTS-GC was conducted based on this edition; pStage II includes pT2 (muscularis propria [MP] and subserosa [SS]) N1 and pT3 (perforates serosa [SE]) N0, pStage IIIA includes pT2N2, pT3N1, and pT4 (invades adjacent structures [SI]) N0, and pStage IIIB includes pT3N2and pT4N1.

OS was estimated in months from the time of surgery to death or the last follow-up assessment date. RFS was estimated in months from the time of surgery to recurrence or death or the last follow- 
up assessment date. Follow-up assessments were performed every 3-6 months for the first 5 years after surgery. Follow-up procedures included medical history, physical examination, routine blood tests, upper endoscopy, and abdominal and pelvic CT. Local recurrence and distant metastases were confirmed histologically or radiographically.

Toxic effects were evaluated according to the Common Terminology Criteria for Adverse Events v4.0.

\section{Statistical Analysis}

Data were analyzed with SPSS statistical software (Chicago, IL, USA). Patient characteristics were compared by two-tailed Fisher's exact test or the $\chi^{2}$ test with Yates correction. Quantitative variables were compared by Student's $t$ test and expressed as median and interquartile range (IQR). Survival times were calculated using the Kaplan-Meier method and compared by the log-rank test. The cutoff point of the prognostic nutritional index (PNI; [10 $\times$ albu$\min ]+[0.005 \times$ total lymphocyte count $]$ ) and N/L ratio (neutrophil count/lymphocyte count) were defined as a Youden index using the receiver-operating characteristics (ROC) curve calculated by each factor as a test variable and OS a state variable. The cutoff points of preoperative $\mathrm{PNI}$, preoperative $\mathrm{N} / \mathrm{L}$ ratio, postoperative PNI, and postoperative N/L ratio were 43.9, 1.56, 36.9, and 1.99, respectively. For the analysis of predictive factors for the completion of 8 courses of S-1 administration, we used the following factors: age ( $<75$ years vs. $\geq 75$ years), sex (female vs. male), American Society of Anesthesiologists physical status ([ASA-PS] 1, 2, or 3), preoperative PNI (<43.6 vs. $\geq 43.9$ ), preoperative Glasgow prognostic score ([GPS] 0,1 , or 2 ), preoperative $\mathrm{N} / \mathrm{L}$ ratio ( $<1.56$ vs. $\geq 1.56$ ), body mass index ([BMI $]<25$ vs. $\geq 25$ ), neoadjuvant chemotherapy (presence vs. absence), type of gastrectomy (distal vs. total), operative approach (open surgery vs. laparoscopy), time to initiation of S-1 administration after surgery ( $<42$ vs. $\geq 42$ days), postoperative complications (Clavien-Dindo $\leq$ grade II vs. $\geq$ grade III), postoperative PNI (<36.9 vs. $\geq 36.9$ ), postoperative GPS $(0,1$, or 2$)$, postoperative $\mathrm{N} / \mathrm{L}$ ratio $(<1.99$ vs. $\geq 1.99)$, creatinine clearance before $S-1$ administration ( $<60 \mathrm{vs.} \geq 60 \mathrm{~mL} / \mathrm{min}$ ), body weight loss (before surgery to 1 month after surgery: $<15$ vs. $\geq 15 \%$ ), and pStage (II, IIIA, or IIIB). Logistic regression analysis for independent predictive factors was performed by inserting all factors listed above.

For the analysis of prognostic factors for OS and RFS, we added the following factors to those used in the analysis of predictive factors for completion of S-1 administration: duration of S-1 administration ( $<4$ vs. $\geq 4$ courses) and completion of S- 1 administration (presence vs. absence). Independent prognostic factors for OS and RFS were identified by Cox proportional regression analysis with the insertion of factors with $p<0.1$ on univariate analysis.

$p<0.05$ was considered statistically significant. The median follow-up time was 56.3 (IQR 31.3-62.2) months.

\section{Results}

\section{Patient Characteristics}

These are listed in Table 1. Median age was 74 years, 6 patients were $\geq 80$ years of age, and $70 \%$ were male. The percentage of patients with ASA-PS category 3 was $17.5 \%$.
Table 1. Characteristics $(n=80)$

\begin{tabular}{|c|c|}
\hline Age, years & $74(72-77)$ \\
\hline Male & $56(70)$ \\
\hline Female & $24(30)$ \\
\hline \multicolumn{2}{|l|}{ ASA-PS } \\
\hline 1 & $27(33.8)$ \\
\hline 2 & $39(48.8)$ \\
\hline 3 & $14(17.5)$ \\
\hline \multicolumn{2}{|l|}{ Preoperative PNI } \\
\hline$<43.9$ & $24(30)$ \\
\hline$\geq 43.9$ & $56(70)$ \\
\hline \multicolumn{2}{|l|}{ Preoperative GPS } \\
\hline 0 & $57(71.3)$ \\
\hline 1 & $17(21.3)$ \\
\hline 2 & $6(7.5)$ \\
\hline \multicolumn{2}{|l|}{ Preoperative N/L } \\
\hline$<1.56$ & $20(25)$ \\
\hline$\geq 1.56$ & $60(75)$ \\
\hline BMI & $22.8(20.5-24.5)$ \\
\hline \multicolumn{2}{|l|}{ Neoadjuvant chemotherapy } \\
\hline Present & $17(21.3)$ \\
\hline \multicolumn{2}{|l|}{ Type of gastrectomy } \\
\hline Distal & $33(41.3)$ \\
\hline Total & $47(58.8)$ \\
\hline \multicolumn{2}{|l|}{ Operative approach } \\
\hline Open & $74(92.5)$ \\
\hline Laparoscopic & $6(7.5)$ \\
\hline Operation time, min & $236.5(200.3-289.3)$ \\
\hline Blood loss, $\mathrm{mL}$ & $355.0(190.0-635.3)$ \\
\hline Postoperative complications (CD > grade III) & $20(25)$ \\
\hline \multicolumn{2}{|l|}{ Postoperative PNI } \\
\hline$<36.9$ & $5(6.3)$ \\
\hline$\geq 36.9$ & $75(93.8)$ \\
\hline \multicolumn{2}{|l|}{ Postoperative GPS } \\
\hline 0 & $49(61.3)$ \\
\hline 1 & $27(33.8)$ \\
\hline 2 & $4(5.0)$ \\
\hline \multicolumn{2}{|l|}{ Postoperative N/L } \\
\hline$<1.99$ & $45(56.3)$ \\
\hline$\geq 1.99$ & $35(43.8)$ \\
\hline \multicolumn{2}{|l|}{ Creatinine clearance } \\
\hline $\begin{array}{l}\text { Before } \mathrm{S}-1 \text { administration, } \mathrm{mL} / \mathrm{min} \\
<60 \mathrm{~mL} / \mathrm{min}\end{array}$ & $\begin{array}{l}68.6(59.1-79.5) \\
58(72.5)\end{array}$ \\
\hline $\begin{array}{l}<60 \mathrm{~mL} / \mathrm{min} \\
\geq 60 \mathrm{~mL} / \mathrm{min}\end{array}$ & $\begin{array}{l}58(72.5) \\
22(27.5)\end{array}$ \\
\hline \multicolumn{2}{|l|}{ Body weight loss } \\
\hline Before surgery to 1 month after surgery, $\%$ & $8.7(6.8-12.4)$ \\
\hline$<15 \%$ & $71(88.8)$ \\
\hline$\geq 15 \%$ & $9(11.3)$ \\
\hline
\end{tabular}

Values express $n$ (\%) or median (IQR). ASA-PS, American Society of Anesthesiologists physical status; PNI, prognostic nutritional index $=$ $(10 \times$ albumin $)+(0.005 \times$ total lymphocyte count $)$; GPS, Glasgow prognostic score; N/L, neutrophil count/lymphocyte count; CD, Clavien-Dindo classification.

Neoadjuvant chemotherapy was administered to $17 \mathrm{pa}-$ tients (docetaxel + cisplatin + S-1 [DCS] to 12 and S- $1+$ cisplatin [SP] to 5). Neoadjuvant chemotherapy was limited to the clinical trial setting. Laparoscopic gastrectomy
Kunisaki et al. 
Table 2. Univariate analysis of predictive factors for completion of 8 courses of S-1 administration

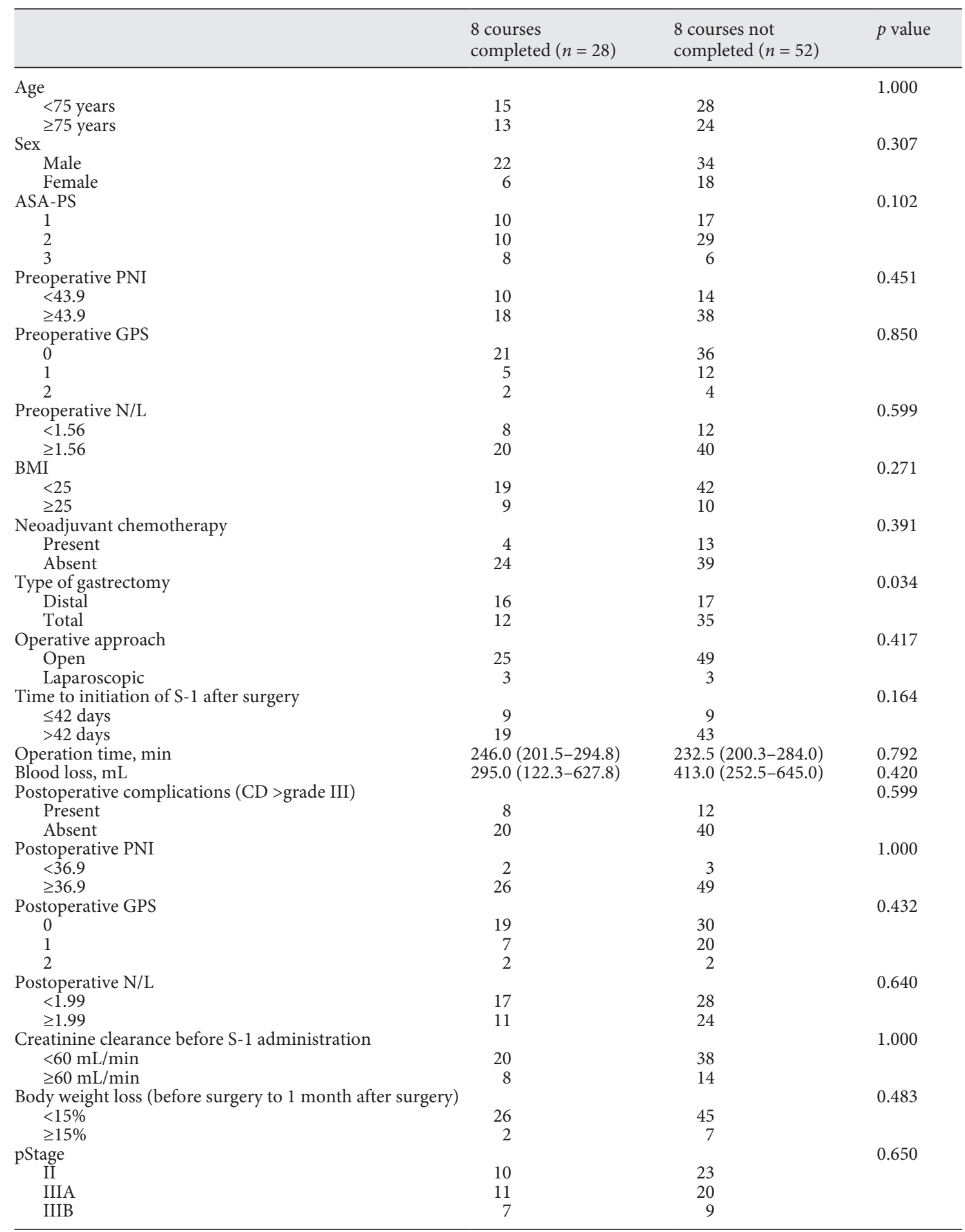

Values express $n$ or median (IQR). ASA-PS, American Society of Anesthesiologists physical status; PNI, prognostic nutritional index $=(10 \times$ albumin $)+(0.005 \times$ total lymphocyte count $)$; GPS, Glasgow prognostic score; N/L, neutrophil count/ lymphocyte count, CD, Clavien-Dindo classification. 
was performed on 6 patients. Postoperative complications $\geq$ grade III were observed in 20 patients, and a body weight loss $\geq 15 \%$ was observed in 9 patients.

\section{Pathological Findings}

The tumors were predominantly located in the lower third of the stomach (36.3\%), followed by the upper third (28.7\%), the middle third (18.8\%), and the entire stomach (16.3\%). Macroscopically ill-defined type tumors (77.5\%) and larger tumors $(<50 \mathrm{~mm}, 35.0 \%$; $\geq 50$ to $<100 \mathrm{~mm}$, $43.8 \%$; $\geq 100 \mathrm{~mm}, 21.3 \%)$ were frequently observed. The numbers of histologically differentiated and undifferentiated type tumors were equal. The median number of retrieved lymph nodes was 37 (IQR 26-44), and the median number of metastatic lymph nodes was 4 (IQR 1-9). pT2, pT3, and pT4 were observed in 51.2, 45.0, and 3.8\% of tumors, respectively. Lymph node metastasis was detected in $92.5 \%$ of tumors. The percentage of pStage II, IIIA, and IIIB tumors was $41.3,38.8$, and $20 \%$, respectively.

\section{S-1 Treatment}

Twenty-eight of 80 patients (35\%) completed 8 courses of S-1. The median time to initiation of S- 1 after surgery was 50 days and S- 1 was administered to 18 patients (22.5\%) within 42 days. The median duration of S- 1 administration was 26.8 weeks; S- 1 was administered for $>16$ weeks (half of the planned dose) to 52 patients. The median relative dose intensity was $82.1 \%$ (IQR 31.1$100 \%)$. Initially, 72 patients (90\%) were started with 4 weeks of S-1 followed by 2 weeks of rest; 17 patients (23.6\%) were converted to 2 weeks of S-1 followed by 1 week of rest. Due to their poor general status, the remaining 8 patients (10\%) were started with 2 weeks of S-1 followed by 1 week of rest. One-step dose reduction was performed in 12 patients (16.7\%), and two-step dose reduction was performed in 2 patients $(2.8 \%)$, principally due to grade III/IV hematological adverse events or grade II/ III/IV nonhematological adverse events, according to the physician's judgment.

\section{Adverse Events during S-1 Treatment}

Regarding $\geq$ grade III hematological adverse events, neutropenia was observed in $8.75 \%$, anemia in $3.75 \%$, thrombocytopenia in $2.5 \%$, and febrile neutropenia in $1.25 \%$ of the patients. Regarding $\geq$ grade II nonhematological adverse events, anorexia and general fatigue were observed in $7.5 \%$, followed by diarrhea in $5 \%$, nausea in $3.75 \%$, pneumonia in $1.25 \%$, and unconsciousness in $1.25 \%$ of the patients. No patient died due to adverse events.
Of 52 patients without completion of S-1 administration, S-1 administration was discontinued in 19 patients (36.5\%) with disease recurrence. Of the remaining $33 \mathrm{pa}-$ tients (63.5\%), 32 discontinued S-1 due to nonhematological toxicity and 6 due to hematological toxicity. A total of $84.3 \%$ patients who suffered from nonhematological toxicity refused the continuation of S-1.

\section{Univariate and Multivariate Analyses of Predictive}

Factors for Completion of S-1 Administration

Univariate analysis showed that the type of gastrectomy was a significant predictive factor for completion of S-1 administration, although the other factors were not selected among 20 factors. Distal gastrectomy significantly predicted completion of S-1 administration (Table 2). Logistic regression analysis also showed that distal gastrectomy independently predicted the completion of S-1 administration (odds ratio [OR] 0.364; 95\% confidence interval $[\mathrm{CI}] 0.141-0.939 ; p=0.037)$.

\section{Univariate and Multivariate Analyses of Prognostic \\ Factors for OS}

Univariate analysis showed that postoperative N/L ratio and pStage significantly influenced OS. A higher postoperative N/L ratio and a more advanced pStage adversely affected OS (Table 3). Multivariate analysis also showed that postoperative N/L ratio (hazard ratio [HR] 2.334; 95\% CI 1.620-5.135; $p=0.035$ ), pStage IIIA (HR 9.362; 95\% CI 2.137-41.01), and pStage IIIB, 11.07; 95\% CI 2.372-51.372; $p<0.001)$ independently influenced OS. Higher postoperative N/L ratio and more advanced stage adversely influenced OS.

\section{Univariate and Multivariate Analyses of Prognostic}

\section{Factors for RFS}

Univariate analysis showed that higher postoperative $\mathrm{N} / \mathrm{L}$ ratio and more advanced stage significantly affected RFS (Table 4). Multivariate analysis also showed that higher postoperative N/L ratio (HR 2.289; 95\% CI 1.0304.895; $p=0.047$ ), pStage IIIA (HR 7.513; 95\% CI 2.81525.83), and pStage IIIB (HR 11.511; 95\% CI 3.191-41.53; $p<0.001)$ were independent prognostic factors for RFS. Higher postoperative N/L ratio and more advanced stage adversely influenced RFS.

\section{Survival}

The 3-year OS and RFS rates were $74.6 \pm 3.2 \%$ and 70.8 $\pm 3.3 \%$, respectively, in all of the 218 registered patients. There were no significant differences between patients who received and those who did not receive S-1 therapy
Kunisaki et al. 
Table 3. Univariate and multivariate analyses of prognostic factors for OS

\begin{tabular}{|c|c|c|c|c|c|}
\hline & $n$ & $\begin{array}{l}\text { Univariate HR } \\
(95 \% \text { CI })\end{array}$ & $p$ value & $\begin{array}{l}\text { Multivariate HR } \\
(95 \% \text { CI })\end{array}$ & $p$ value \\
\hline Age & & & 0.605 & & \\
\hline$<75$ years & 43 & 1 & & & \\
\hline$\geq 75$ years & 37 & $1.221(0.573-2.600)$ & & & \\
\hline Sex & & & 0.217 & & \\
\hline Female & 24 & 1 & & & \\
\hline Male & 56 & $1.844(0.698-4.874)$ & & & \\
\hline ASA-PS & & & 0.206 & & \\
\hline 1 & 27 & 1 & & & \\
\hline 2 & 39 & $0.487(0.205-1.159)$ & & & \\
\hline 3 & 14 & $1.026(0.385-2.738)$ & & & \\
\hline Preoperative PNI & & & 0.111 & & \\
\hline$<43.9$ & 24 & 1 & & & \\
\hline$\geq 43.9$ & 56 & $2.202(0.833-5.818)$ & & & \\
\hline Preoperative GPS & & & 0.627 & & \\
\hline 0 & 57 & 1 & & & \\
\hline 1 & 17 & $1.270(0.533-3.024)$ & & & \\
\hline 2 & 6 & $0.471(0.063-3.520)$ & & & \\
\hline Preoperative N/L & & & 0.174 & & \\
\hline$<1.56$ & 20 & 1 & & & \\
\hline$\geq 1.56$ & 60 & $2.090(0.723-6.047)$ & & & \\
\hline BMI & & & 0.342 & & \\
\hline$<25$ & 61 & 1 & & & \\
\hline$\geq 25$ & 19 & $0.624(0.236-1.650)$ & & & \\
\hline Neoadjuvant chemotherapy & & & 0.693 & & \\
\hline Absent & 63 & 1 & & & \\
\hline Present & 17 & $1.201(0.484-2.977)$ & & & \\
\hline Type of gastrectomy & & & 0.969 & & \\
\hline Distal & 33 & 1 & & & \\
\hline Total & 47 & $1.015(0.475-2.172)$ & & & \\
\hline Operative approach & & & 0.284 & & \\
\hline Open & 74 & 1 & & & \\
\hline Laparoscopic & 6 & $0.043(0-14.14)$ & & & \\
\hline Time to initiation of S- 1 after surgery & & & 0.276 & & \\
\hline$\leq 42$ days & 26 & 1 & & & \\
\hline$>42$ days & 54 & $0.653(0.303-1.407)$ & & & \\
\hline Duration of S-1 administration & & & 0.572 & & \\
\hline$<16$ weeks & 28 & 1 & & & \\
\hline$\geq 16$ weeks & 52 & $0.798(0.365-1.745)$ & & & \\
\hline Postoperative complications & & & & & \\
\hline (CD > grade III) & & & 0.787 & & \\
\hline Absent & 60 & 1 & & & \\
\hline Present & 20 & $0.882(0.356-2.187)$ & & & \\
\hline Postoperative PNI & & & 0.460 & & \\
\hline$<36.9$ & 5 & 1 & & & \\
\hline$\geq 36.9$ & 75 & $2.125(0.288-15.67)$ & & & \\
\hline Postoperative GPS & & & & & \\
\hline 0 & 49 & 1 & & & \\
\hline 1 & 27 & $1.004(0.443-2.275)$ & & & \\
\hline 2 & 4 & $2.072(0.474-9.056)$ & & & \\
\hline Postoperative N/L & & & 0.006 & & 0.035 \\
\hline$<1.99$ & 45 & 1 & & 1 & \\
\hline$\geq 1.99$ & 35 & $2.991(1.365-6.552)$ & & $2.334(1.061-5.135)$ & \\
\hline Creatinine clearance before $\mathrm{S}-1$ administration & & & 0.224 & & \\
\hline$<60 \mathrm{~mL} / \mathrm{min}$ & 58 & 1 & & & \\
\hline$\geq 60 \mathrm{~mL} / \mathrm{min}$ & 22 & $0.547(0.207-1.445)$ & & & \\
\hline Body weight loss (before surgery to 1 month after & r surgery) & & 0.924 & & \\
\hline$<15 \%$ & 71 & 1 & & & \\
\hline$\geq 15 \%$ & 9 & $0.943(0.284-3.134)$ & & & \\
\hline pStage & & & 0.004 & & $<0.001$ \\
\hline II & 33 & 1 & & 1 & \\
\hline IIIA & 31 & $10.63(2.439-46.28)$ & & $9.362(2.137-41.01)$ & \\
\hline IIIB & 16 & $12.83(2.767-59.51)$ & & $11.07(2.372-51.372)$ & \\
\hline Completion of S-1 administration & & & 0.279 & & \\
\hline Absent & 52 & 1 & & & \\
\hline Present & 28 & $0.633(0.277-1.448)$ & & & \\
\hline
\end{tabular}

OS, overall survival; HR, hazard ratio; CI, confidence interval; ASA-PS, American Society of Anesthesiologists physical status; PNI, prognostic nutritional index $=(10 \times$ albumin $)+(0.005 \times$ total lymphocyte count $)$; GPS, Glasgow prognostic score; N/L, neutrophil count/lymphocyte count; CD, Clavien-Dindo classification. 
Table 4. Univariate and multivariate analyses of prognostic factors for RFS

\begin{tabular}{|c|c|c|c|c|c|}
\hline & $n$ & $\begin{array}{l}\text { Univariate HR } \\
(95 \% \text { CI })\end{array}$ & $p$ value & $\begin{array}{l}\text { Multivariate HR } \\
(95 \% \text { CI })\end{array}$ & $p$ value \\
\hline Age & & & 0.946 & & \\
\hline$<75$ years & 43 & 1 & & & \\
\hline$\geq 75$ years & 37 & $1.025(0.500-2.102)$ & & & \\
\hline Sex & & & 0.836 & & \\
\hline Female & 24 & 1 & & & \\
\hline Male & 56 & $1.089(0.485-2.447)$ & & & \\
\hline ASA-PS & & & 0.327 & & \\
\hline 1 & 27 & 1 & & & \\
\hline 2 & 39 & $0.561(0.251-1.252)$ & & & \\
\hline 3 & 14 & $0.962(0.365-2.332)$ & & & \\
\hline Preoperative PNI & & & 0.330 & & \\
\hline$<43.9$ & 24 & 1 & & & \\
\hline$\geq 43.9$ & 56 & $1.523(0.653-3.553)$ & & & \\
\hline Preoperative GPS & & & 0.957 & & \\
\hline 0 & 57 & 1 & & & \\
\hline 1 & 17 & $1.128(0.479-2.654)$ & & & \\
\hline 2 & 6 & $0.951(0.233-4.058)$ & & & \\
\hline Preoperative N/L & & & 0.103 & & \\
\hline$<1.56$ & 20 & 1 & & & \\
\hline$\geq 1.56$ & 60 & $2.398(0.837-6.872)$ & & & \\
\hline BMI & & & 0.510 & & \\
\hline$<25$ & 61 & 1 & & & \\
\hline$\geq 25$ & 19 & $0.740(0.302-1.811)$ & & & \\
\hline Neoadjuvant chemotherapy & & & 0.208 & & \\
\hline Absent & 63 & 1 & & & \\
\hline Present & 17 & $1.682(0.748-3.781)$ & & & \\
\hline Type of gastrectomy & & & 0.573 & & \\
\hline Distal & 33 & 1 & & & \\
\hline Total & 47 & $1.234(0.593-2.567)$ & & & \\
\hline Operative approach & & & 0.256 & & \\
\hline Open & 74 & 1 & & & \\
\hline Laparoscopic & 6 & $0.043(0-9.780)$ & & & \\
\hline Time to initiation of S- 1 after surgery & & & 0.221 & & \\
\hline$\leq 42$ days & 26 & 1 & & & \\
\hline$>42$ days & 54 & $0.634(0.305-1.316)$ & & & \\
\hline Duration of S- 1 administration & & & 0.316 & & \\
\hline$<16$ weeks & 28 & 1 & & & \\
\hline$\geq 16$ weeks & 52 & $0.668(0.331-1.429)$ & & & \\
\hline Postoperative complications (CD > grade III) & & & 0.665 & & \\
\hline Absent & 60 & 1 & & & \\
\hline Present & 20 & $1.196(0.532-2.687)$ & & & \\
\hline Postoperative PNI & & & 0.379 & & \\
\hline$<36.9$ & 5 & 1 & & & \\
\hline$\geq 36.9$ & 75 & $2.449(0.333-17.99)$ & & & \\
\hline Postoperative GPS & & & 0.827 & & \\
\hline 0 & 49 & 1 & & & \\
\hline 1 & 27 & $0.987(0.455-2.140)$ & & & \\
\hline 2 & 4 & $1.564(0.362-6.757)$ & & & \\
\hline Postoperative N/L & & & 0.016 & & 0.047 \\
\hline$<1.99$ & 45 & 1 & & 1 & \\
\hline$\geq 1.99$ & 35 & $2.454(1.280-5.110)$ & & $2.289(1.030-4.895)$ & \\
\hline Creatinine clearance before S-1 administration & & & 0.109 & & \\
\hline$<60 \mathrm{~mL} / \mathrm{min}$ & 58 & 1 & & & \\
\hline$\geq 60 \mathrm{~mL} / \mathrm{min}$ & 22 & $0.445(0.174-1.190)$ & & & \\
\hline Body weight loss (before surgery to 1 month after & urgery) & & 0.778 & & \\
\hline$<15 \%$ & 71 & 1 & & & \\
\hline$\geq 15 \%$ & 9 & $0.842(0.255-2.778)$ & & & \\
\hline pStage & & & 0.001 & & 0.001 \\
\hline II & 33 & 1 & & 1 & \\
\hline IIIA & 31 & $7.513(2.185-25.83)$ & & $7.513(2.185-25.83)$ & \\
\hline IIIB & 16 & $11.51(3.191-41.53$ & & $11.511(3.191-41.53)$ & \\
\hline Completion of S-1 administration & & & 0.117 & & \\
\hline Absent & 52 & 1 & & & \\
\hline Present & 28 & $0.541(0.240-1.215)$ & & & \\
\hline
\end{tabular}

RFS, relapse-free survival; HR, hazard ratio; CI, confidence interval; ASA-PS, American Society of Anesthesiologists physical status; PNI, prognostic nutritional index $=(10 \times$ albumin $)+(0.005 \times$ total lymphocyte count $)$; GPS, Glasgow prognostic score; N/L, neutrophil count/lymphocyte count. 

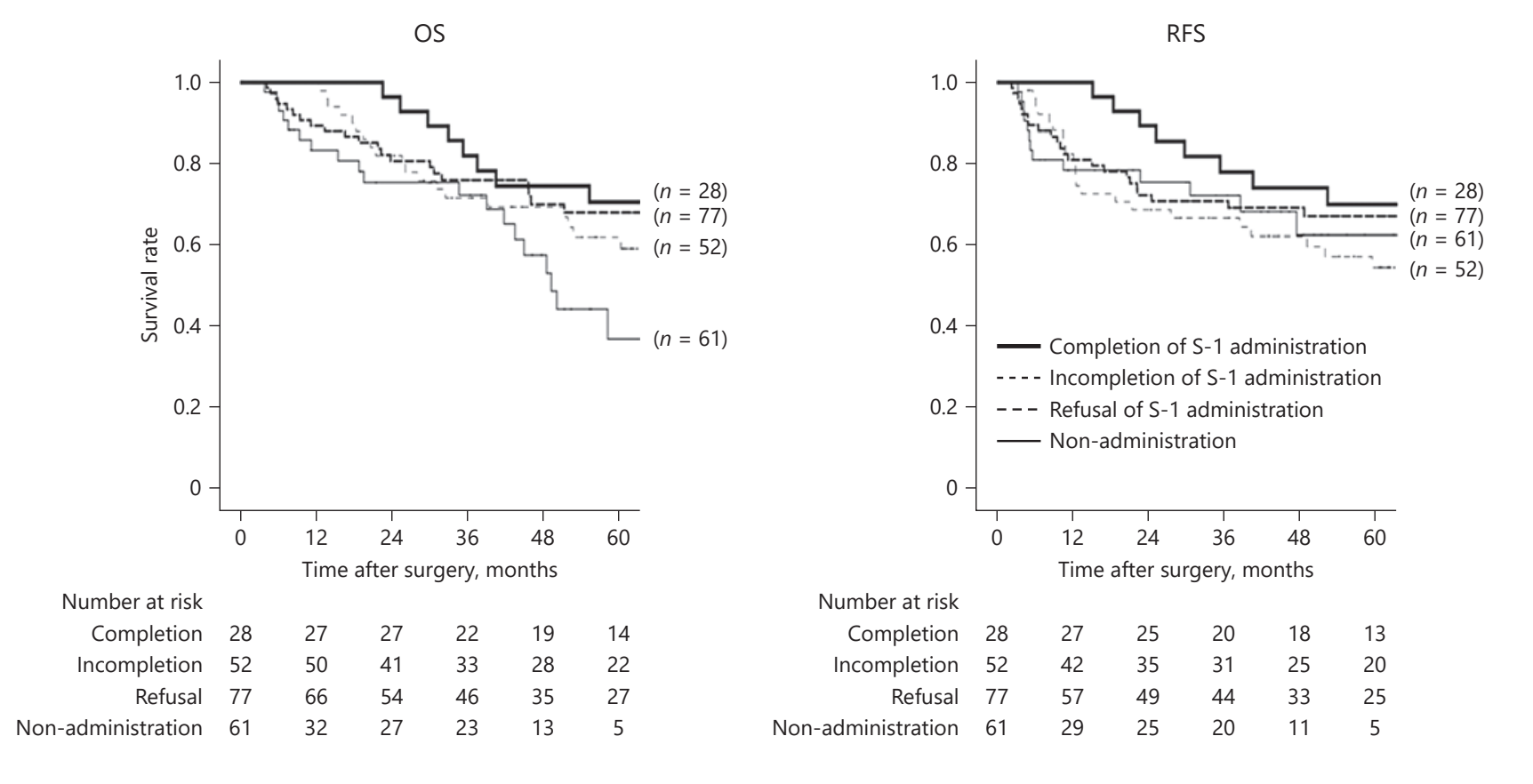

Fig. 1. Analysis of overall survival (OS) and relapse-free survival (RFS) in all registered patients. There were significant differences in OS between the completion group and the nonadministration group (3-year survival rate: $82.0 \pm 7.3 \%$ vs. $72.3 \pm 7.2 \% ; p=0.025)$, and between the refusal group and the nonadministration group ( 3 -year survival rate: $76.0 \pm 5.1 \%$ vs. $72.3 \pm 7.2 \%$; $p=0.041$ ). However, there were no significant differences in RFS between each group.

regarding OS $(75.3 \pm 4.9 \%$ vs. $74.6 \pm 4.2 \% ; p=0.284)$ and RFS (70.6 \pm 5.2 vs. $71.3 \pm 4.3 \% ; p=0.945)$ at 3 years.

There were no significant differences in OS and RFS between the 2 groups according to each stage. There were significant differences in OS between the completion group and the nonadministration group (3-year survival rate: $82.0 \pm 7.3 \%$ vs. $72.3 \pm 7.2 \%$; $p=0.025$ ), and between the refusal group and the nonadministration group (3year survival rate: $76.0 \pm 5.1 \%$ vs. $72.3 \pm 7.2 \% ; p=0.041$ ) However, there were no significant differences in RFS between each group (Fig. 1).

Among pStage II patients, there were significant differences in OS between the incompletion group and the refusal group (3-year survival rate: $100 \pm 0 \%$ vs. $87.4 \pm$ $5.3 \%$; $p=0.038$ ), and the nonadministration group (3year survival rate: $100 \pm 0 \%$ vs. $78.3 \pm 8.6 \%$; $p=0.001$ ). Furthermore, OS differed significantly in the completion group and the nonadministration group (3-year survival rate: $90 \pm 9.5 \%$ vs. $78.3 \pm 8.6 \% ; p=0.049$ ). Among pStage IIIA patients, there was a significant difference in OS between patients who completed and those who did not complete S-1 therapy (3-year survival rate: $90.9 \pm 8.7 \%$ vs.
$49.1 \pm 11.4 \% ; p=0.024$ ), but no significant differences were found between patients who completed and those who refused S-1 therapy (3-year survival rate: $59.8 \pm$ $11.2 \%$ ), or who did not undergo S-1 therapy (3-year survival rate: $60.9 \pm 13.8 \%)$. Among pStage IIIB patients, we did not find any difference in OS between the incompletion group (55.6 $\pm 16.6 \%)$, the completion group (57.1 \pm $18.7 \%)$, the refusal group $(62.7 \pm 15.5 \%)$, and the nonadministered group ( $83.8 \pm 15.2 \%)$ at 3 years (Fig. 2 ).

Among pStage II patients, there were no significant differences in RFS between the incompletion group ( 95.5 $\pm 4.4 \%)$, the completion group $(90.0 \pm 9.5 \%)$, the refusal group $(92.2 \pm 4.3 \%)$, and the nonadministered group $(90.9 \pm 6.1 \%)$ at 3 years. We found a significant difference in RFS between pStage IIIA patients who completed and those who did not complete S-1 therapy (3-year survival rate: $80.8 \pm 12.2 \%$ vs. $50.0 \pm 11.2 \%$; $p=0.039$ ). However, no significant differences in RFS were found between patients who completed and those who did refused S- 1 therapy (3-year survival rate: $56.6 \pm 11.0 \%$ ), or who did not undergo S-1 therapy (3-year survival rate, $55.6 \pm 13.6 \%$ ). Among pStage IIIB patients, no significant differences 


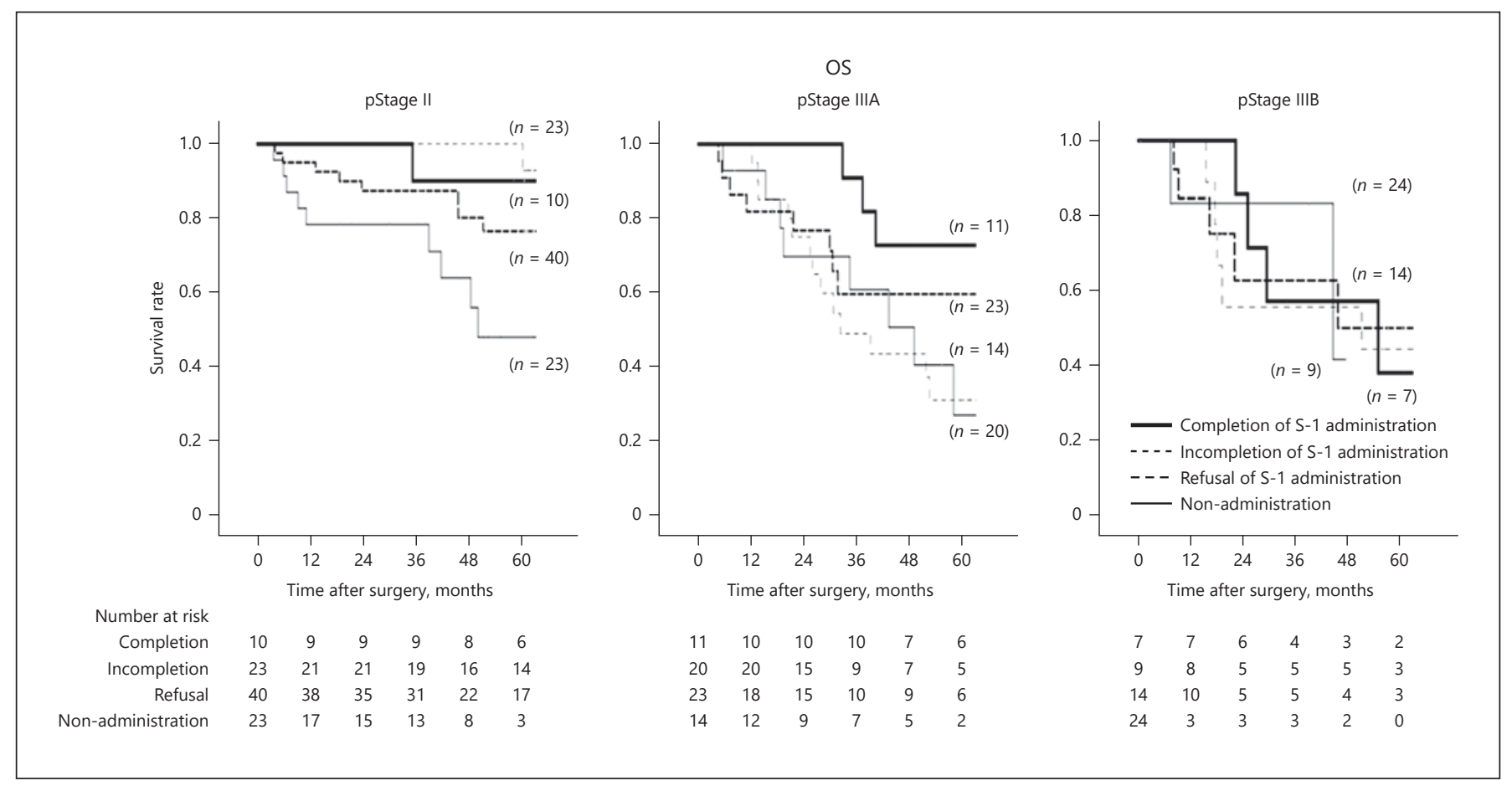

Fig. 2. Analysis of overall survival by stage. Among pStage II patients, there were significant differences in OS between the incompletion group and the refusal group (3-year survival rate: $100 \pm 0 \%$ vs. $87.4 \pm 5.3 \% ; p=0.038$ ), and the nonadministration group (3year survival rate: $100 \pm 0 \%$ vs. $78.3 \pm 8.6 \%$; $p=0.001$ ). Furthermore, OS showed significant difference between the completion

were observed in RFS between the incompletion group $(33.3 \pm 15.7 \%)$, the completion group $(57.1 \pm 18.7 \%)$, the refusal group $(27.5 \pm 13.5 \%)$, and the nonadministered group $(50.0 \pm 20.4 \%)$ at 3 years (Fig. 3$)$.

\section{Cause of Death and Pattern of Recurrence}

Twenty-eight patients died during the study, including 23 who died of gastric cancer. Lymphogenous metastasis was detected in 10 patients (extraregional lymph node metastasis in 8), peritoneal dissemination in 9, hematogenous metastasis in 5 , and pleuritis carcinomatosa in 2 . Of the remaining 5 patients, 1 died of hepatocellular carcinoma, 2 died of other diseases, and 2 died due to natural causes. Three living patients with recurrence ( 2 cases of lymphogenous metastasis and 1 of peritoneal dissemination) were treated by chemotherapy during the study.

\section{Treatment after Recurrence}

Of the 26 patients with cancer recurrence, 19 (73.1\%) underwent second-line chemotherapy and 7 received best supportive care. Of the 19 receiving chemotherapy, a pa- group and the nonadministration group (3-year survival rate: $90 \pm$ $9.5 \%$ vs. $78.3 \pm 8.6 \%$; $p=0.049$ ). Among pStage IIIA patients, there was a significant difference in OS between patients who completed and those who did not complete S-1 therapy (3-year survival rate: $90.9 \pm 8.7 \%$ vs. $49.1 \pm 11.4 \%$; $p=0.024)$. Among pStage IIIB patients, we did not find any difference in OS between each group.

clitaxel-based regimen was administered in 8 , a docetaxel-based regimen in 6, and an S-1-based regimen in 5 (26.3\%). Of these 19 patients receiving chemotherapy, 5 were moved to third-line treatment ( 3 to a CPT-11-based regimen, 1 to a paclitaxel-based regimen, and 1 to a docetaxel-based regimen). No patient was moved to a fourth-line regimen, but 1 underwent radiotherapy for para-aortic lymph node metastasis and achieved complete remission without recurrence.

\section{Discussion}

This retrospective study showed that distal gastrectomy independently predicted completion of S-1 administration. Higher postoperative N/L ratio and more advanced pStage adversely influenced OS and RFS independently.

In 2007, the ACTS-GC trial showed that S-1 monotherapy was a useful adjuvant regimen for stage II/III gastric cancer patients after D2 gastrectomy [6]. In 2011, the
48

Eur Surg Res 2021;62:40-52 DOI: $10.1159 / 000515175$
Kunisaki et al. 


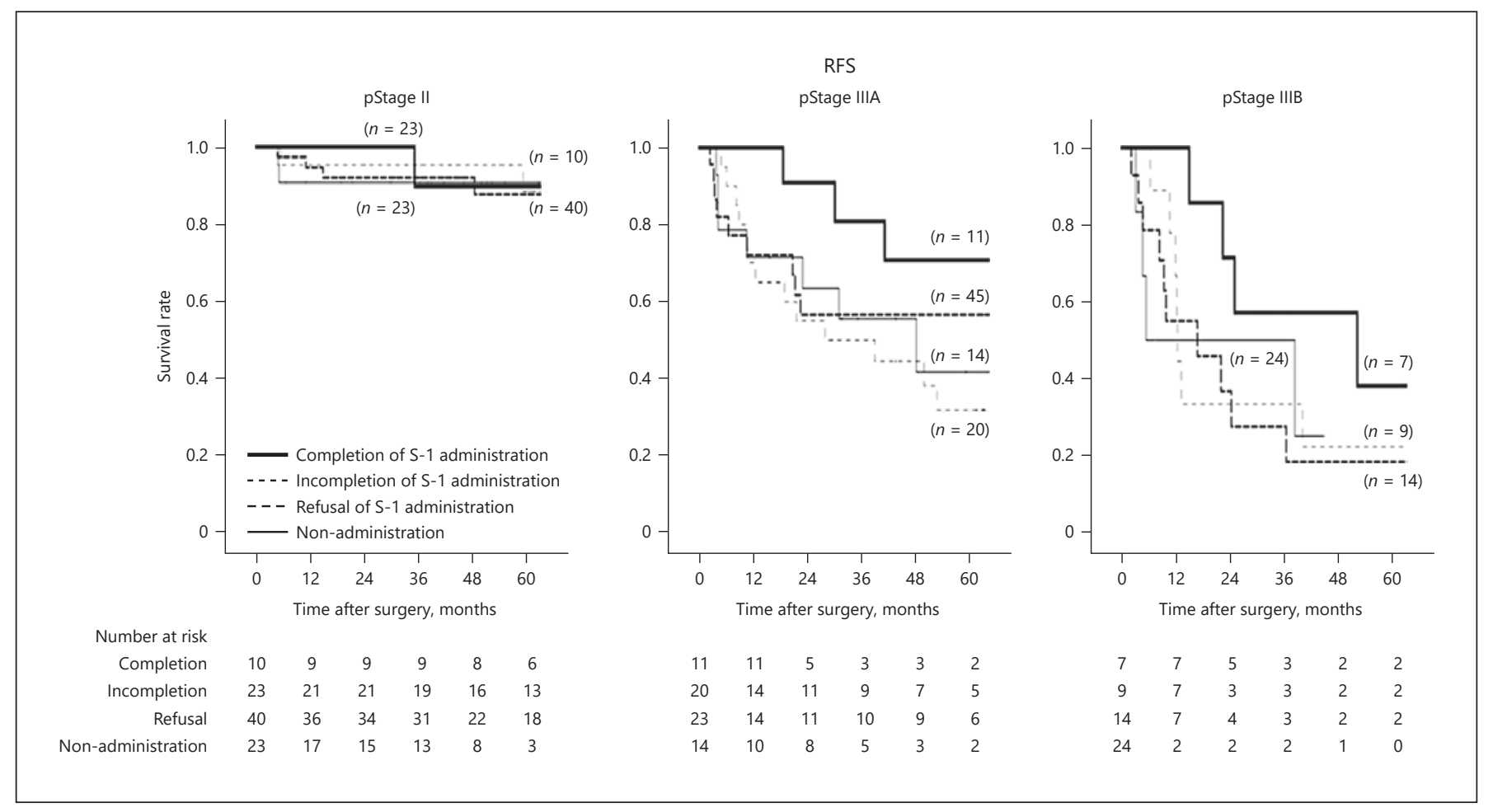

Fig. 3. Analysis of relapse-free survival (RFS) by stage. Among pStage II patients, there were no significant differences in RFS between each group. We found a significant difference in RFS between pStage IIIA patients who completed and those who did not complete S-1 therapy (3-year survival rate: $80.8 \pm 12.2 \%$ vs. $50.0 \pm 11.2 \%$; $p=$ 0.039). Among pStage IIIB patients, no significant differences were observed in RFS between each group.

5-year follow-up data confirmed these favorable results [7]. Therefore, administration of 8 courses of adjuvant S-1 was regarded as a standard treatment for stage II/III gastric cancer after R0 resection. However, in a subset of the population, it is difficult to start S-1 administration within 6 weeks after surgery and to complete S-1 administration over 1 year. Total and partial gastrectomy reduce digestion and absorption capabilities, resulting in less oral intake and poor nutritional status. Particularly in elderly patients, total gastrectomy and postoperative infectious complications lead to malnutrition and thus adversely affect the continuation of S-1 administration [14]. In this study, we focused on elderly gastric cancer patients, since the proportion of such patients will increase in the future. There have been few reports concerning the efficacy of adjuvant S-1 monotherapy in elderly patients with pStage II/III gastric cancer $[17,18]$ although there are some studies focusing on the outcome of S-1 monotherapy for advanced gastric cancer $[19,20]$. It is difficult to analyze the data due to the variety of therapeutic strategies and outcomes of elderly patients, as a standard adjuvant treatment cannot be uniformly administered in this population. Here, we evaluated the results of S-1 monotherapy as adjuvant chemotherapy for gastric cancer in the elderly to present real-world data from a highvolume center in Yokohama, Japan.

Preoperative comorbidity and postoperative complications made it difficult to start S-1 administration in the very elderly patients. Therefore, it is necessary to meticulously take care of such patients and prevent postoperative complications by performing more delicate operative procedures.

A Korean study reported that total gastrectomy was an independent predictive factor for severe adverse events with S-1 adjuvant chemotherapy [21]. In our study, although we did not evaluate total gastrectomy as a predictive factor for severe adverse events, total gastrectomy was selected as an independent predictive factor for not completing S-1 administration. As severe adverse events can be a cause of incompletion of S-1 administration, our results confirm those of the Korean study. Compared to distal gastrectomy, total gastrectomy severely reduces amounts of oral intake and induces poor nutrition in the elderly. As a result, it becomes difficult to continue the administration 
of S-1 in a subset of the elderly population. It is important to preserve the stomach as much as possible based on the oncological standpoint. The Korean study also discussed the start time of S-1 administration for elderly gastric cancer patients undergoing total gastrectomy. They argued that it may be safer to initiate S-1 administration at lower doses and then increase the dose if it is tolerated. S-1 is commonly initiated at a standard dose, but it is necessary to pay attention to renal function. Thereafter, we usually reduce the dosage of S-1 when severe adverse events are observed. It is necessary to determine the appropriate strategy for initiating the dose in elderly gastric cancer patients, particularly those undergoing total gastrectomy, in a prospective randomized controlled study. In this study, we did not find significant differences in both OS and RFS between distal and total gastrectomy although distal gastrectomy independently predicted completion of S- 1 administration. Total gastrectomy actually diminishes quality of life of patients compared to partial gastrectomy [22], and so medical intervention in addition to nutritional support should be available. However, some other factors like preoperative immunonutritional status and pathological stage robustly affect survival. Therefore, distal gastrectomy itself does not influence survival. In this study, completion of S-1 administration was not selected an independent prognostic factor for OS and RFS. It is easily supposed that S-1 administration did not affect OS as elderly patients died due to diseases other than gastric cancer. However, the small number of patients in this study may have influenced the result that there was no influence of completion of S-1 administration for RFS. Therefore, it is mandatory to evaluate this important issue in many patients.

In this study, $36.7 \%$ of pStage II/III gastric cancer patients accepted the planned adjuvant S-1 chemotherapy. Thus, about $35.3 \%$ of patients rejected the chemotherapy in spite of sufficient explanation and $28.0 \%$ of patients were judged as having no indications for S-1 administration. Many elderly patients may reject adjuvant chemotherapy because they lose confidence after surgery and require all their energy to recover. S- 1 administration was started within 42 days after gastrectomy in only $32.5 \%$ of patients. This result suggests that many elderly patients cannot recover from the surgical burden within 42 days. It is important to identify the optimal start time of S-1 administration by considering the general status of each patient. Moreover, $35 \%$ of patients could not continue S- 1 oral intake for $>4$ courses. A previous study showed that survival time was significantly worse in pStage II gastric cancer patients receiving 4 courses of S- 1 administration than in those receiving 8 courses [8]. We should therefore make an effort to continue S-1 administration as long as possible.

In this study, 28 of 230 (12.2\%) elderly patients had an indication for S-1 adjuvant chemotherapy and the planned dose of S-1 was successfully administered to 28 of 80 (35.0\%) elderly patients actually receiving S-1 chemotherapy. However, the completion rate in this study was lower than that in previous studies that included all age groups $[6,14]$. Therefore, it is necessary to perform certain arrangements to complete S-1 administration in the elderly.

In the 5-year outcomes of the ACTS-GC study [7], OS and RFS were significantly improved in the S-1 administration group compared to group that underwent surgery only in stage II and IIIA. By contrast, S-1 administration did not improve OS and RFS compared to the refusal group and the nonadministration group in any stage. The ACTS-GC study is a prospective one with many patients under the strict indication. Our study was a retrospective one with a small number of patients including the many categories of elderly patients. However, OS and RFS in the completion of S-1 administration group showed favorable outcomes compared to the other groups with pStage IIIA. Therefore, it is necessary to ascertain which elderly patients will tolerate the treatment and take measures like dose reduction and longer intervals, in order to complete S-1 administration in this population.

The rates of both $\geq$ grade III hematological and $\geq$ grade II nonhematological adverse events were similar to those in the ACTS-GC trials. Prophylactic and aggressive dose reduction and conversion to administration for 2 weeks followed by 1 week of rest resulted in acceptable rates of adverse events, even in the elderly.

Cox proportional hazards regression analysis showed that higher postoperative N/L ratio and more advanced pStage independently influenced OS in this study. Higher postoperative N/L ratio was also selected as an independent prognostic factor, but preoperative N/L ratio was not. A previous study similarly reported the impact of postoperative N/L ratio on prognosis [23]. However, other studies focusing on preoperative N/L ratio reported that $\mathrm{N} / \mathrm{L}$ ratio was an independent prognostic factor in gastric cancer [24-26]. Immunological status may change due to the surgical burden, including postoperative complications, and so the postoperative N/L ratio accurately represents an immunological status that may support tumor growth by regulating the microenvironment of residual cancer cells, despite gastrectomy. Higher postoperative N/L ratio was also an independent prognostic factor for RFS in this study.
Kunisaki et al. 
It is important to focus on the postoperative $\mathrm{N} / \mathrm{L}$ ratio and to examine whether a more effective adjuvant chemotherapy regimen, such as a doublet regimen, would be useful to improve long-term survival in elderly patients with pStage II/III gastric cancer. There has been no study evaluating the long-term outcomes of a combination adjuvant therapy for pStage II/III gastric cancer in the elderly. In contrast, some clinical groups have studies comparing the therapeutic outcomes between S-1 monotherapy and S-1/cisplatin therapy for advanced gastric cancer in the elderly $[27,28]$. They showed the survival benefits of S-1/cisplatin therapy with a high incidence of adverse events.

We have to pay attention to adverse events when combination therapy is performed in the elderly. Recent phase II and III studies reported the efficacy of combination adjuvant chemotherapy for pStage II/III gastric cancer irrespective of age. Many clinicians employ S-1 monotherapy for stage II gastric cancer and apply S-1/oxaliplatin [29], capecitabine/oxaliplatin [30], and docetaxel/S-1 [31] therapies for stage III gastric cancer as adjuvant chemotherapy in Japan. However, we do not have data about the feasibility of these regimens for elderly gastric cancer patients. A feasibility study on a large sample of patients to confirm the usefulness of these regimens for the elderly is warranted.

Cox proportional hazards regression analysis also revealed that more advanced pStage was an independent prognostic factor for both OS and RFS. pStage has a strong impact on survival in elderly gastric cancer patients. In this study, completion of S-1 administration was an independent prognostic factor for RFS. This result is compatible with previous studies [32]. It is essential to complete 8 courses of S- 1 administration to obtain satisfactory long-term outcomes.

The 5-year OS and RFS in this study were equivalent to rates in the middle range between patients with and without S-1 administration in the ACTS-GC trial. As the ACTS-GC trial includes all age groups, the outcomes obtained in our study on elderly patients only may be worse. We need to arrange the administration protocol to improve survival in elderly gastric cancer patients.

There are some limitations to this retrospective study. First, the number of patients was small. However, the number of elderly patients who receive S-1 adjuvant chemotherapy is low, and it is therefore necessary to conduct a multicenter study to involve many patients. Second, the therapeutic decision when adverse events were observed was dependent on the individual judgment of each physician, who decided to either reduce the dose or change the duration (and intervals between doses) of administration in the early phase of the study. It will be necessary to standardize the indication and strategy for adverse events.

In a previous study, medical oncologists could facilitate completion of 1 year of S-1 irrespective of patients' age more effectively than surgeons. Medical oncologists may offer sufficient explanations and arrange adequate dose reduction and duration of S-1 administration for each patient. These efforts resulted in favorable long-term outcomes. Therefore, it is important to make various kinds of efforts to obtain satisfactory therapeutic benefits by completing S-1 administration in elderly gastric cancer patients. A phase III trial (JCOG 1507) to confirm S-1 adjuvant chemotherapy for pStage II/III vulnerable elderly gastric cancer patients ( $\geq 80$ years) is now ongoing in Japan. The results will hopefully provide us with the optimal strategy for S-1 adjuvant chemotherapy in the elderly.

To complete S-1 administration as adjuvant setting in elderly patients with pStage II/III gastric cancer, total gastrectomy should be avoided if possible. A new regimen for elderly gastric cancer patients with higher postoperative N/L ratios and more advanced stage should be established.

\section{Statement of Ethics}

This study was approved by the Institutional Review Board (approval No. B2007000028) and conformed to the principles of the Declaration of Helsinki. As this is retrospective study, the informed consent was obtained according to the concept of opt out displayed on the website of the university.

\section{Conflict of Interest Statement}

C.K. had the following conflicts of interest: Taiho Pharmaceutical Co. Ltd., Chugai Pharmaceutical Co. Ltd., and Yakult Honsha Co. Ltd. The other authors have no conflicts of interest to declare.

\section{Funding Sources}

There was no funding.

\section{Author Contributions}

C.K. and I.E.: study concept and manuscript editing; C.K.: study design and statistical analysis; S.S., N.T., K.K., and T.K.: data acquisition; S.T. and T.K.: quality control of data and algorithms; H.A. and J.W.: data analysis and interpretation; C.K. and S.S.: manuscript preparation; all authors: manuscript review. 


\section{References}

1 Forman D, Burley VJ. Gastric cancer: global pattern of the disease and an overview of environmental risk factors. Best Pract Res Clin Gastroenterol. 2006;20(4):633-49.

2 Fitzmaurice A, Allen C, Barber RM, Barregard L, Bhutta ZA, Brenner H, et al. Global, Regional, and National Cancer Incidence, Mortality, Years of Life Lost, Years Lived with Disability, and Disability-Adjusted Life-years for 32 Cancer Groups, 1990 to 2015: A Systematic Analysis for the Global Burden of Disease Study. JAMA Oncol. 2017;3:524-48.

3 Ludwig K, Möller D, Bernhardt J. Surgical management for early stage gastric cancer. Chirurg. 2018 May;89(5):347-57. German.

4 Nishizawa T, Yahagi N. Long-term outcomes of using endoscopic submucosal dissection to treat early gastric cancer. Gut Liver. 2018 Mar; 12(2):119-24.

5 Fuchs CS, Mayer RJ. Gastric carcinoma. N Engl J Med. 1995 Jul;333(1):32-41.

6 Sakuramoto S, Sasako M, Yamaguchi T, Kinoshita T, Fujii M, Nashimoto A, et al.; ACTS-GC Group. Adjuvant chemotherapy for gastric cancer with S-1, an oral fluoropyrimidine. N Engl J Med. 2007 Nov;357(18): 1810-20.

7 Sasako M, Sakuramoto S, Katai H, Kinoshita T, Furukawa H, Yamaguchi T, et al. Five-year outcomes of a randomized phase III trial comparing adjuvant chemotherapy with S-1 versus surgery alone in stage II or III gastric cancer. J Clin Oncol. 2011 Nov;29(33):438793.

8 Yoshikawa T, Terashima M, Mizusawa J, Nunobe S, Nishida Y, Yamada T, et al. Four courses versus eight courses of adjuvant S-1 for patients with stage II gastric cancer (JCOG1104 [OPAS-1]): an open-label, phase 3 , non-inferiority, randomised trial. Lancet Gastroenterol Hepatol. 2019 Mar;4(3):20816.

9 Kang YK, Chin K, Chung HC, Kadowaki S, Oh SC, Nakayama N, et al. S-1 plus leucovorin and oxaliplatin versus $\mathrm{S}-1$ plus cisplatin as first-line therapy in patients with advanced gastric cancer (SOLAR): a randomised, openlabel, phase 3 trial. Lancet Oncol. 2020 Aug; 21(8):1045-56.

10 Boku N, Ryu MH, Kato K, Chung HC, Minashi K, Lee KW, et al. Safety and efficacy of nivolumab in combination with S-1/ capecitabine plus oxaliplatin in patients with previously untreated, unresectable, advanced, or recurrent gastric/gastroesophageal junction cancer: interim results of a randomized, phase II trial (ATTRACTION-4). Ann Oncol. 2019 Feb;30(2):250-8.

11 Yong WP, Rha SY, Tan IB, Choo SP, Syn NL, Koh V, et al.; Singapore Gastric Cancer Consortium (SGCC). Real-Time Tumor Gene Expression Profiling to Direct Gastric Can- cer Chemotherapy: Proof-of-Concept " $3 G$ ” Trial. Clin Cancer Res. 2018 Nov;24(21): 5272-81

12 Yamamoto $\mathrm{N}$, Hayashi $\mathrm{H}$, Planchard $\mathrm{D}$, Morán T, Gregorc V, Dowell J, et al. A randomized, phase 2 study of deoxyuridine triphosphatase inhibitor, TAS-114, in combination with S-1 versus S-1 alone in patients with advanced non-small-cell lung cancer. Invest New Drugs. 2020 Oct;38(5):1588-97.

13 Winther SB, Liposits G, Skuladottir H, Hofsli E, Shah CH, Poulsen L $\varnothing$, et al. Reduced-dose combination chemotherapy (S-1 plus oxaliplatin) versus full-dose monotherapy (S-1) in older vulnerable patients with metastatic colorectal cancer (NORDIC9): a randomised, open-label phase 2 trial. Lancet Gastroenterol Hepatol. 2019 May;4(5):376-88.

14 Yamashita K, Kurokawa Y, Yamamoto K, Hirota M, Kawabata R, Mikami J, et al. Risk factors for poor compliance with adjuvant S-1 chemotherapy for gastric cancer: A multicenter retrospective study. Ann Surg Oncol. 2017 Sep;24(9):2639-45.

15 Kano Y, Ohashi M, Hiki N, Takahari D, Chin $\mathrm{K}$, Yamaguchi K, et al. Facilitated completion of 1-year adjuvant S-1 monotherapy for pathological stage II or III gastric cancer by medical oncologists. Surg Today. 2020 Oct;50(10): 1197-205.

16 Japanese Gastric Cancer Association. Japanese Classification of Gastric Carcinoma -2nd English Edition. Gastric Cancer. 1998 Dec; 1(1):10-24.

17 Tsushima T, Hironaka S, Boku N, Machida N, Yamazaki K, Yasui H, et al. Safety and efficacy of S-1 monotherapy in elderly patients with advanced gastric cancer. Gastric Cancer. 2010 Nov;13(4):245-50.

18 Aoyama T, Yoshikawa T, Watanabe T, Hayashi T, Ogata T, Cho H, et al. Safety and feasibility of S-1 adjuvant chemotherapy for gastric cancer in elderly patients. Gastric Cancer. 2012 Jan;15(1):76-82.

19 Bando H, Yamada Y, Tanabe S, Nishikawa K, Gotoh M, Sugimoto N, et al. Efficacy and safety of S-1 and oxaliplatin combination therapy in elderly patients with advanced gastric cancer. Gastric Cancer. 2016 Jul;19(3):919-26.

20 Makiyama A, Kunieda K, Noguchi M, Kajiwara T, Tamura T, Takeda K, et al. First-line chemotherapy with S-1 alone or S-1 plus cisplatin for elderly patients with advanced gastric cancer: a multicenter propensity scorematched study. Gastric Cancer. 2018 Sep; 21(5):792-801.

21 Jeong JH, Ryu MH, Ryoo BY, Lee SS, Park I, Lee SH, et al. Safety and feasibility of adjuvant chemotherapy with S-1 for Korean patients with curatively resected advanced gastric cancer. Cancer Chemother Pharmacol. 2012 Oct; 70(4):523-9.
22 Kwon OK, Yu B, Park KB, Park JY, Lee SS, Chung HY. Advantages of Distal Subtotal Gastrectomy over Total Gastrectomy in the Quality of Life of Long-Term Gastric Cancer Survivors. J Gastric Cancer. 2020 Jun;20(2) 176-89.

23 Min KW, Kwon MJ, Kim DH, Son BK, Kim EK, Oh YH, et al. Persistent elevation of postoperative neutrophil-to-lymphocyte ratio: A better predictor of survival in gastric cancer than elevated preoperative neutrophil-tolymphocyte ratio. Sci Rep. 2017 Oct;7(1): 13967.

24 Jung MR, Park YK, Jeong O, Seon JW, Ryu SY, Kim DY, et al. Elevated preoperative neutrophil to lymphocyte ratio predicts poor survival following resection in late-stage gastric cancer. J Surg Oncol. 2011 Oct;104(5):504-10.

25 Graziosi L, Marino E, De Angelis V, Rebonato A, Cavazzoni E, Donini A. Prognostic value of preoperative neutrophils to lymphocytes ratio in patients resected for gastric cancer. Am J Surg. 2015 Feb;209(2):333-7.

26 Borda A, Vila J, Fernández-Urién I, Zozaya JM, Guerra A, Borda F. Pretreatment predictive value of blood neutrophil/lymphocyte ratio in R0 gastric cancer resectability. Gastroenterol Hepatol. 2017 Jan;40(1):1-9.

27 Terazawa T, Iwasa S, Takashima A, Nishitani $\mathrm{H}$, Honma Y, Kato K, et al. Impact of adding cisplatin to S-1 in elderly patients with advanced gastric cancer. J Cancer Res Clin Oncol. 2013 Dec;139(12):2111-6.

28 Sasaki Y, Iwasa S, Okazaki S, Goto M, Kojima Y, Naganuma A, et al. A phase II study of combination therapy with oral S-1 and cisplatin in elderly patients with advanced gastric cancer. Gastric Cancer. 2018 May;21(3):439_ 45.

29 Koizumi W, Takiuchi H, Yamada Y, Boku N, Fuse N, Muro K, et al. Phase II study of oxaliplatin plus S-1 as first-line treatment for advanced gastric cancer (G-SOX study). Ann Oncol. 2010 May;21(5):1001-5.

30 Fuse N, Bando H, Chin K, Ito S, Yoshikawa T, Tsuburaya A, et al. Adjuvant capecitabine plus oxaliplatin after D2 gastrectomy in Japanese patients with gastric cancer: a phase II study. Gastric Cancer. 2017 Mar;20(2):332-40.

31 Yoshida K, Kodera Y, Kochi M, Ichikawa W, Kakeji Y, Sano T, et al. Addition of docetaxel to oral fluoropyrimidine improves efficacy in patients with stage III gastric cancer: interim analysis of JACCRO GC-07, a randomized controlled trial. J Clin Oncol. 2019 May; 37(15):1296-304

32 Miyatani K, Saito H, Shimizu S, Kono Y, Murakami Y, Shishido $Y$, et al. Late start and insufficient S-1 dose in adjuvant chemotherapy can lead to poor prognosis in stage II/III gastric cancer. Int J Clin Oncol. 2019 Oct;24(10): 1190-6. 